Research Paper

\title{
Concurrent Chemoradiotherapy versus Intensity- modulated Radiotherapy Alone for Elderly Nasopharyngeal Carcinoma Patients with Pre-treatment Epstein-Barr Virus DNA: A Cohort Study in an Endemic Area with Long-term Follow-up
}

\author{
Qin Yang1,2, Ting-Ting Zhao 3 , Meng-Yun Qiang1,4, Liang Hu1,5, Xing Lv1,5, Yan-Fang Ye6, Liang-Ru Ke1,5, \\ Ya-Hui Yu1,5, Wen-Ze Qiu1,5, Guo-Ying Liu1,5, Xin-Jun Huang1,5, Wang-Zhong Li1,5, Shu-Hui Lv'1,5, Yao \\ Sun7, Lu-Yao Zhang8, Fei Pei ${ }^{8}$, Xiang Guo 1,5, Yan-Qun Xiang1,5, Chao-Nan Qian ${ }^{1,5 \bowtie}$, Bi-Jun Huang1 ${ }^{\circledR}$, \\ Wei-Xiong Xia ${ }^{1,5}$ \\ 1. Collaborative Innovation Center for Cancer Medicine, State Key Laboratory of Oncology in South China, Sun Yat-Sen University Cancer Center, \\ Guangzhou, China. \\ 2. Department of Gynecology Oncology, Shaanxi Provincial Cancer Hospital Affiliated to Medical School, Xi'an Jiaotong University, Xi'an, China. \\ 3. Department of Oncology, Guiyang Hospital of Guizhou Aviation Industry Group, Guizhou, China. \\ 4. Department of Radiotherapy, Sun Yat-Sen University Cancer Center, Guangzhou, China. \\ 5. Department of Nasopharyngeal Carcinoma, Sun Yat-Sen University Cancer Center, Guangzhou, China. \\ 6. Clinical Trial Design Division, Clinical Research Center, Sun Yat-Sen Memorial Hospital, Sun Yat-Sen University, Guangzhou, China. \\ 7. The Third Affiliated Hospital of Sun Yat-Sen University, Sun Yat-Sen University, Guangzhou, China. \\ 8. The First Affiliated Hospital of Sun Yat-Sen University, Sun Yat-Sen University, Guangzhou, China.
}

$\triangle$ Corresponding author: Chao-Nan Qian, MD, PhD, Bi-Jun Huang, PhD, and Wei-Xiong Xia, MD, PhD, Department of Nasopharyngeal Carcinoma, Sun Yat-Sen University Cancer Center, 651 Dongfeng Road East, Guangzhou, 510060, P. R. China. E-mail: qianchn@sysucc.org.cn, huangbj@sysucc.org.cn, and xiawx@sysucc.org.cn; Tel.: +86-20-87343392; Fax: +86-20-87343359

(C) Ivyspring International Publisher. This is an open access article distributed under the terms of the Creative Commons Attribution (CC BY-NC) license (https://creativecommons.org/licenses/by-nc/4.0/). See http://ivyspring.com/terms for full terms and conditions.

Received: 2018.03.18; Accepted: 2018.06.09; Published: 2018.07.30

\begin{abstract}
Purpose: To date, no guidelines exist for elderly nasopharyngeal carcinoma (NPC) patients (60 years of age or older) due to a lack of prospective clinical trials. This study evaluated the efficacy of concurrent chemotherapy (CCRT) for NPC in elderly patients treated with intensity-modulated radiotherapy (IMRT).

Methods: Patients were identified from a prospectively maintained database. A total of 198 consecutive cases of elderly patients with NPC receiving IMRT, including 103 patients treated with IMRT plus CCRT and 95 patients treated with IMRT alone, were analysed from January 2002 to December 2013. Multivariate analysis (MVA) using the Cox proportional hazards model and propensity score analysis (PSA) were performed for overall survival (OS) and disease-free survival (DFS). Finally, sensitivity analysis was performed.

Results: The median follow-up time was 55.3 months (range, 3-135.6 months). In the entire cohort, both MVA and PSA models showed that compared with IMRT alone, IMRT plus CCRT significantly improved survival (hazard ratio [HR] 2.143, 95\% confidence interval [95\% Cl] 1.180-3.890; HR 1.961, 95\% Cl, 1.117-3.443, for OS and DFS, respectively). Similar results were found in the subgroups with high levels of Epstein-Barr virus (EBV) DNA, except in the low-EBV-DNA cohort. The total rates of severe acute toxicity, including leukopenia, neutropenia, stomatitis, and emesis, were significantly higher in the IMRT+CCRT group than in the IMRT-alone group $(P<0.001)$ but were similar to the rates of severe late toxicity $(P=0.818)$. Sensitivity analysis confirmed the robustness of our analysis.
\end{abstract}

Conclusions: In the era of IMRT, CCRT retained survival benefits at high EBV DNA levels but not at low EBV DNA levels for elderly NPC patients. Randomized clinical trials are needed to confirm our findings.

Key words: elderly nasopharyngeal carcinoma, concurrent chemotherapy, intensity-modulated radiotherapy, EBV DNA, propensity score analysis 


\section{Introduction}

Nasopharyngeal carcinoma (NPC) differs from other head and neck cancers because of its unique characteristics in terms of epidemiology, aetiology, clinical presentation, and prognostic factors [1, 2]. NPC is fairly prevalent in Southern China and Southeast Asia [3-5], with a peak incidence from 50 to 60 years of age. Elderly NPC patients (60 years of age or older) account for approximately $14 \%$ of all NPC cases. The currently recommended standard regimen is the addition of concurrent chemotherapy (CCRT) for NPC patients who receive radiotherapy (RT) with or without adjuvant chemotherapy, although this approach carries higher rates of acute toxicity.

However, treatment guidelines are principally tailored for non-elderly patients, and elderly NPC patients are commonly excluded from clinical trials because of restrictive selection criteria. Several previous studies have shown that CCRT is feasible and effective and could achieve reasonable local control in elderly NPC patients receiving RT [6, 7]. Unfortunately, these studies were based on elderly patients treated with 2DRT. The latest studies have reported that adding CCRT did not improve the survival of NPC patients receiving intensity-modulated radiotherapy (IMRT) [8, 9]. Moreover, elderly patients receiving CCRT might be more prone to the accompanying complications and in inferior physical condition and would thus suffer more serious toxicities [10]. Therefore, in the era of IMRT, it remains unknown whether CCRT is necessary and provides a survival benefit for elderly patients.

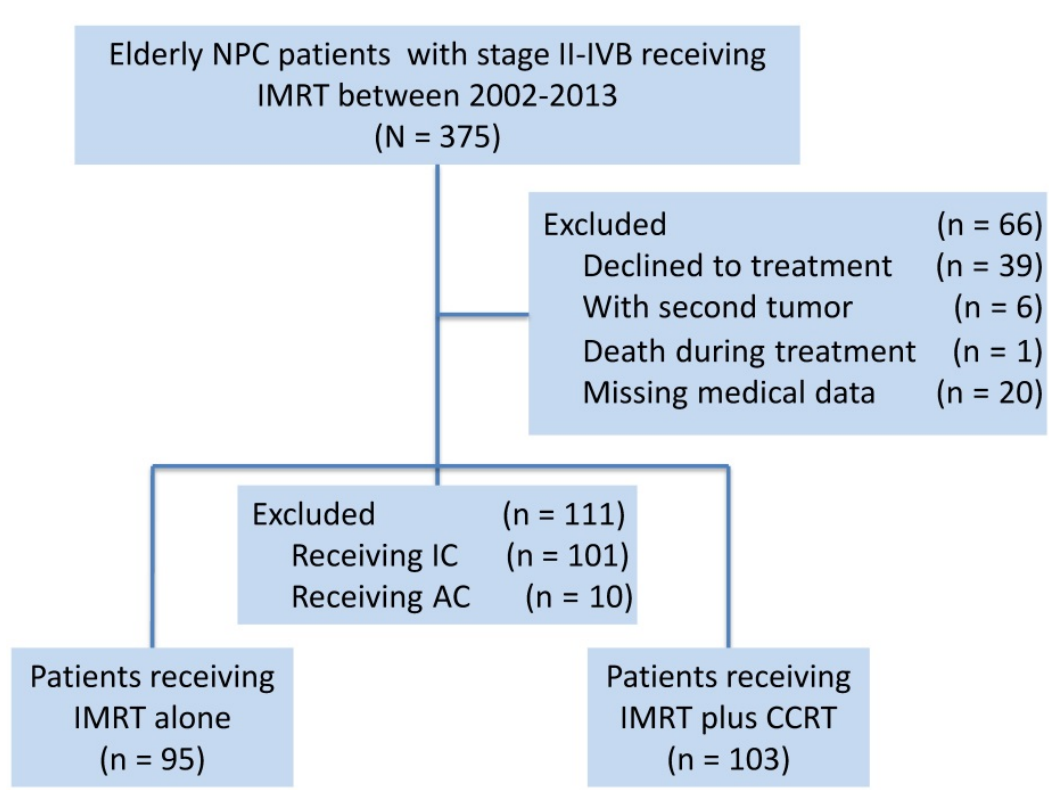

Figure 1. Diagram of the analytic cohort for survival analysis. Abbreviations: AC, adjuvant chemotherapy; CCRT, concurrent chemotherapy; IC, induction chemotherapy; IMRT, intensity-modulated radiotherapy.
We therefore investigated long-term cohorts from an endemic area to assess whether elderly patients benefit from CCRT compared to the outcomes of IMRT alone.

\section{Materials and methods}

\section{Patients and ethics statement}

Based on a prospectively created database, this study selected consecutive elderly patients (age equal to or greater than 60 years) who were receiving radical treatment with IMRT at Sun Yat-Sen University Cancer Center (SYSUCC) between January 2002 and December 2013. A total of 375 newly diagnosed cases of NPC, stage II-IVB, restaged using the seventh edition of the AJCC/UICC staging system, were identified. The following exclusion criteria were used: (1) patients who declined treatment $(\mathrm{n}=39)$; (2) patients with a second tumour $(\mathrm{n}=6)$; (3) death during treatment $(\mathrm{n}=1)$; (4) missing medical data $(\mathrm{n}=20)$; (5) patients who received treatment with induction chemotherapy $(n=101)$; and (6) patients who received treatment with adjuvant chemotherapy $(n=10)$. Considering that the survival benefits of induction chemotherapy in NPC patients remain controversial and that induction chemotherapy would confound the ability of this study to evaluate the efficacy of concurrent chemotherapy [1], we excluded patients receiving induction chemotherapy from the study. A total of 177 cases were excluded. Finally, 198 elderly patients over 60 years of age were identified (Figure 1).

This study was reviewed and approved by the Institutional Review Board and Ethics Committee of SYSUCC, Guangzhou, China. All the patient records were anonymized and de-identified prior to analysis.

\section{Real-time quantitative EBV DNA measurement}

Plasma Epstein-Barr virus (EBV) DNA concentrations, as described in a previous report [11], were routinely measured by real-time quantitative polymerase chain reaction (PCR) prior to treatment [12]. The plasma EBV DNA concentration was calculated using the following equation: $C=Q \times$ $(V D N A / V P C R) \times(1 / V E X T)$, in which $C$ represents the target concentration in plasma (copies/mL), $Q$ represents the target quantity (copy number) determined by PCR, VDNA represents the total volume of DNA obtained after extraction (typically $50 \mu \mathrm{L} /$ Qiagen 
extraction), $V P C R$ represents the volume of DNA solution used for PCR (typically $2 \mu \mathrm{L}$ ), and VEXT represents the volume of plasma extracted (typically $0.5 \mathrm{~mL}$ ) [12].

Of 198 patients, 139 patients were measured prior to treatment. Four prespecified low-EBV-DNA groups and two prespecified high-EBV-DNA groups, based on a cut-off of 4,000 copies/mL, were analysed. The selected cut-off level was chosen because this threshold has been shown to be prognostic in previous NPC studies using the same measurement system [13-15].

\section{Treatment with chemotherapy and radiotherapy}

All the NPC patients were treated with IMRT according to a protocol established in previous studies $[9,16]$. One hundred and three patients received IMRT combined with platinum-based CCRT, and 95 patients were treated with IMRT alone. CCRT consisted of concurrent cisplatin weekly (30-40 $\mathrm{mg} / \mathrm{m}^{2}$ ) or $80-100 \mathrm{mg} / \mathrm{m}^{2}$ cisplatin on days 1,22 , and 43 during radiotherapy [17, 18]. Of the patients receiving IMRT plus CCRT, 38 patients $(35.9 \%)$ were treated with platinum administered weekly, and 66 patients $(64.1 \%)$ received chemotherapy consisting of 1 to 3 cycles every 3 weeks (Supplementary Table 1).

\section{Outcome and follow-up}

The primary endpoint was overall survival (OS), which was calculated from the date of initial treatment to the date of death, caused by any reason, or patient censoring at the date of the last follow-up. The secondary endpoint was disease-free survival (DFS), calculated from the date of initial treatment to the date of death or relapse from any cause or patient censoring at the date of the last follow-up.

After treatment completion, patients were examined every three months during the first three years and then every six months thereafter until death. A detailed history and a complete physical examination were performed at each follow-up visit. Specifically, nasopharyngoscopy, magnetic resonance imaging (MRI) scanning of the head and neck, chest radiography, abdominal sonography, whole-body bone scanning using single-photon emission computed tomography (SPECT) or ${ }^{18} \mathrm{~F}$-fluorodeoxyglucose positron emission tomography and computed tomography (PET/CT) were routinely performed annually or when patients had clinical symptoms that indicated tumour relapse.

Chemotherapy-related toxicities were graded using the Common Terminology Criteria for Adverse Events (version 3.0). Radiotherapy-related toxicities were graded by both the Acute and Late Radiation
Morbidity Scoring Criteria of the Radiation Therapy Oncology Group [19]. We characterized tumour responses in accordance with the Response Evaluation Criteria in Solid Tumors [20].

\section{Statistical analysis}

OS and DFS were estimated with the Kaplan-Meier method. The log-rank test was used in the univariate analysis. The Chi-square test, Fisher's exact test, and Student's t-test were used to compare the differences between the IMRT+CCRT group and the IMRT-alone group. Multivariate analysis (MVA) was performed using the Cox proportional hazards model with the forward stepwise method.

Propensity score analysis (PSA) adjusts for the bias introduced by non-random treatment assignment by comparing patients who had a similar likelihood of receiving a treatment but who received different treatments [21]. For this analysis, based on the patients' characteristics, we used a generalized boosting model to calculate the propensity score with the smallest mean square error (MSE). Then, the effect of treatment on survival was estimated with inverse probability weighting, which can adjust for other confounders with better balance and estimate the effect with more precision than matching because of complete samples with maximized power. A marginal Cox proportional hazards model was then applied using maximum partial likelihood estimates of regression parameters and a robust sandwich covariance matrix [22-24].

Finally, we performed sensitivity analysis to measure the potential unmeasured influence to validate the robustness of the analysis results. All statistical tests were two sided, and $\mathrm{P}<0.05$ was considered significant. All statistical analyses were performed in $\mathrm{R}$ (version 2.15.3).

\section{Results}

\section{Patient characteristics}

Characteristics of the total 198 NPC patients, including 103 patients (52\%) receiving IMRT+CCRT and 95 patients (48\%) receiving IMRT alone, are listed in Table 1. The percentages of clinical stages II, III, and IVA-B were $23.7 \%, 48.5 \%$, and $27.8 \%$, respectively. There were no significant differences in the distribution of sex, body mass index (BMI), education, smoking, drinking, Charlson/Deyo comorbidity score, EA-IgA, EBV DNA, or year of diagnosis (all P values > 0.05), but significant differences were observed for age, VCA-IgA, tumour (T) and node (N) stages, and clinical stage. Patients with older age or in earlier stages were more likely to receive IMRT alone than were patients receiving IMRT plus CCRT $(\mathrm{P}<$ $0.05)$. 
Table 1. Baseline of characteristics in the entire cohort

\begin{tabular}{|c|c|c|c|c|}
\hline \multirow[t]{2}{*}{ Characteristics } & \multicolumn{2}{|c|}{ No.(\%) of patients } & \multirow[t]{2}{*}{ Total $(\mathrm{N}=198)$} & \multirow[t]{2}{*}{$P$ value } \\
\hline & $\begin{array}{l}\text { IMRT+CCRT }(n \\
=103,52 \%)\end{array}$ & $\begin{array}{l}\text { IMRT alone (n } \\
=95,48 \%)\end{array}$ & & \\
\hline Age & & & & $<0.001$ \\
\hline Median(SD) & $63(3.7)$ & $67(5.5)$ & $64(4.9)$ & \\
\hline Sex & & & & 0.301 \\
\hline Male & $76(73.8)$ & $76(80.0)$ & $152(76.8)$ & \\
\hline Female & $27(26.2)$ & $19(20.0)$ & $46(23.2)$ & \\
\hline Body mass index & & & & 0.358 \\
\hline$\leq 18.49$ & $8(7.8)$ & $3(3.2)$ & $11(5.6)$ & \\
\hline $18.50-22.99$ & $37(35.9)$ & $42(44.2)$ & $79(39.9)$ & \\
\hline $23.00-27.50$ & $50(48.5)$ & $45(47.4)$ & $95(48.0)$ & \\
\hline$>27.50$ & $8(7.8)$ & $5(5.3)$ & $13(6.6)$ & \\
\hline Education & & & & 0.090 \\
\hline Unschooled & $8(7.8)$ & $7(7.4)$ & $15(7, .6)$ & \\
\hline Low & $43(41.7)$ & $24(25.3)$ & $67(33.8)$ & \\
\hline Middle & $35(34.0)$ & $45(47.4)$ & $80(40.4)$ & \\
\hline High & $17(16.5)$ & $19(20.0)$ & $36(18.2)$ & \\
\hline Smoke & & & & 0.822 \\
\hline Yes & $45(43.7)$ & $40(42.1)$ & $85(42.9)$ & \\
\hline No & $58(56.3)$ & $55(57.9)$ & $113(57.1)$ & \\
\hline Drink & & & & 0.667 \\
\hline Yes & $12(11.7)$ & 13(13.7) & $25(12.6)$ & \\
\hline No & $91(88.3)$ & $82(86.3)$ & $173(87.4)$ & \\
\hline Charlson/Deyo co & brbidity score & & & 0.117 \\
\hline 0 & $61(59.2)$ & $59(62.1)$ & $120(60.6)$ & \\
\hline 1 & 3735.9) & $25(26.3)$ & $62(31.3)$ & \\
\hline $2-$ & $5(4.9)$ & $11(11.6)$ & $16(8.1)$ & \\
\hline VCA-IgA & & & & 0.047 \\
\hline$<1: 80$ & $7(6.8)$ & $14(14.7)$ & $21(10.6)$ & \\
\hline $1: 80-1: 320$ & $61(59.2)$ & $61(64.2)$ & $122(61.6)$ & \\
\hline$\geq 1: 640$ & $35(34.0)$ & $20(21.1)$ & $55(27.8)$ & \\
\hline EA-IgA & & & & 0.078 \\
\hline$<1: 10$ & $17(16.5)$ & $26(27.4)$ & $43(21.7)$ & \\
\hline $1: 10-1: 20$ & $31(30.1)$ & $32(33.7)$ & $63(31.8)$ & \\
\hline$\geq 1: 40$ & $55(53.4)$ & $37(38.9)$ & $92(46.5)$ & \\
\hline EBV-DNA & & & & 0.544 \\
\hline $0-999$ & $32(31.1)$ & $38(40.0)$ & $70(35.4)$ & \\
\hline $1000-9999$ & $16(15.5)$ & $13(13.7)$ & $29(14.6)$ & \\
\hline 10000-99999 & $16(15.5)$ & $9(9.5)$ & $25(12.6)$ & \\
\hline $100000-$ & $9(8.7)$ & $6(6.3)$ & $15(7.6)$ & \\
\hline Unmeasured & $30(29.1)$ & $29(30.5)$ & $59(29.8)$ & \\
\hline Histology, WHO ty & & & & 0.525 \\
\hline II & $4(3.9)$ & $6(6.3)$ & $10(5.1)$ & \\
\hline III & $99(96.1)$ & $89(93.7)$ & $188(94.9)$ & \\
\hline Tumor stage & & & & 0.002 \\
\hline 1 & $4(3.9)$ & $6(6.3)$ & $10(5.1)$ & \\
\hline 2 & $18(17.5)$ & $35(36.8)$ & $53(26.8)$ & \\
\hline 3 & $47(45.6)$ & $40(42.1)$ & $87(43.9)$ & \\
\hline 4 & $34(33.0)$ & $14(14.7)$ & $48(24.2)$ & \\
\hline Node stage & & & & 0.003 \\
\hline 0 & $18(17.5)$ & $38(40.0)$ & $56(28.3)$ & \\
\hline 1 & $43(41.7)$ & $35(36.8)$ & $78(39.4)$ & \\
\hline 2 & $37(35.9)$ & $19(20.0)$ & $56(28.3)$ & \\
\hline 3 & $5(4.9)$ & $3(3.2)$ & $8(4.0)$ & \\
\hline Clinical stage & & & & $<0.001$ \\
\hline II & $12(11.7)$ & $35(36.8)$ & $47(23.7)$ & \\
\hline III & $52(50.5)$ & $44(46.3)$ & $96(48.5)$ & \\
\hline IVA-B & $39(37.9)$ & $16(16.8)$ & $55(27.8)$ & \\
\hline Year of diagnosis & & & & 0.186 \\
\hline $2002-2006$ & $20(19.4)$ & 18(18.9) & $38(19.2)$ & \\
\hline 2007-2009 & $41(39.8)$ & $27(28.4)$ & $68(34.3)$ & \\
\hline $2010-2013$ & $42(40.8)$ & $50(52.6)$ & $92(46.5)$ & \\
\hline
\end{tabular}

Abbreviation: CCRT, concurrent chemotherapy; EBV, Epstein - Barr virus; IMRT, intensity-modulated radiotherapy; SD, standard deviation.

\section{Clinical response}

Table 2 shows the response to treatment in the two groups. At the end of treatment, there was no significant difference in complete response (CR) rates between the IMRT+CCRT and IMRT-alone groups
( $83.5 \%$ vs. $87.4 \%, P=0.441$ ). Specifically, the $C R$ rates at the primary and lymph node sites were $91.3 \%$ and 94.7\% ( $\mathrm{P}=0.412)$ and $90.3 \%$ and $92.6 \%(\mathrm{P}=0.289)$ for patients receiving IMRT+CCRT and patients receiving IMRT alone, respectively. Three months after treatment, the CR rate at the primary site for the IMRT+CCRT and IMRT-alone groups was $97.1 \%$ and $98.9 \%$, respectively $(\mathrm{P}=0.672)$, while this rate was $97.1 \%$ and $95.8 \%$, respectively, at the lymph node site $(\mathrm{P}=0.913)$. Overall, there were similar responses to treatment between the IMRT+CCRT and IMRT-alone groups $(94.2 \%$ vs. $94.7 \%, \mathrm{P}=0.863)$.

Table 2. Response to treatments of IMRT with or without concurrent chemotherapy

\begin{tabular}{|c|c|c|c|c|c|}
\hline \multirow{2}{*}{$\begin{array}{l}\text { Response to } \\
\text { treatment }\end{array}$} & \multicolumn{2}{|c|}{ CCRT+IMRT } & \multicolumn{3}{|c|}{ IMRT alone } \\
\hline & Number & Percent (\%) & Number & Percent $(\%)$ & P value \\
\hline \multicolumn{6}{|c|}{ End of treatment } \\
\hline \multicolumn{6}{|c|}{ Nasopharyngeal site } \\
\hline Residual & 9 & 8.7 & 5 & 5.3 & 0.412 \\
\hline \multicolumn{6}{|c|}{ Lymph node site } \\
\hline Residual & 10 & 9.7 & 5 & 5.3 & 0.289 \\
\hline Stable & 0 & 0 & 2 & 2.1 & 0.229 \\
\hline Total any & 17 & 16.5 & 12 & 12.6 & 0.441 \\
\hline \multicolumn{6}{|c|}{$\begin{array}{l}\text { Three months after } \\
\text { treatment }\end{array}$} \\
\hline \multicolumn{6}{|c|}{ Nasopharyngeal site } \\
\hline Residual & 3 & 2.9 & 1 & 1.1 & 0.672 \\
\hline \multicolumn{6}{|c|}{ Lymph node site } \\
\hline Residual & 3 & 2.9 & 4 & 4.2 & 0.913 \\
\hline Total any & 6 & 5.8 & 5 & 5.3 & 0.863 \\
\hline
\end{tabular}

\section{Survival outcomes}

With a median follow-up of 55.3 months (range, 3.0-135.6 months), the 5-year OS and DFS rates were $75.3 \%$ and $72.3 \%$, respectively. The 5-year OS and DFS rates of elderly patients with clinical stage II, III, or IVA-B were $92.7 \%, 77.6 \%$, and $56.2 \%$, respectively, and $89.7 \%, 73.5$, and $55.0 \%$, respectively. For patients receiving IMRT plus CCRT, the 5-year OS and DFS rates were $74.7 \%$ and $73.8 \%$, respectively, while these rates were $76.2 \%$ and $70.6 \%$ for patients treated with IMRT alone, respectively.

Table 3 shows the results of the MVA by Cox proportional hazards model. After the analysis was adjusted for observational factors, compared with patients in the IMRT+CCRT group, patients in the IMRT-alone group showed a significantly higher mortality risk for OS (adjusted hazard ratio [HR] $1.934,95 \%$ confidence interval [95\% CI], 1.085-3.450, P $=0.025)$ and DFS (HR 2.511, 95\% CI, 1.240-5.088, $\mathrm{P}=$ $0.011)$.

\section{PSA by inverse probability of treatment weighting}

As a part of the sensitivity analysis, to confirm the MVA results and to recalculate the effects of the 
two treatment regimens using various algorithms via reducing confounding, a propensity score weighting model was performed with observational confounders. The results of PSA were similar to those of the MVA with the Cox proportional hazards model (Table 3).

Table 3. Multivariable Analysis of the Primary Cohort

\begin{tabular}{|c|c|c|c|c|}
\hline \multirow[t]{2}{*}{ Characteristics } & \multicolumn{2}{|l|}{ OS } & \multicolumn{2}{|l|}{ DFS } \\
\hline & HR(95\%CI) & $\mathrm{P}$ & $\mathrm{HR}(95 \% \mathrm{CI})$ & $P$ value \\
\hline \multicolumn{5}{|l|}{ PSA/IPTW* } \\
\hline \multicolumn{5}{|c|}{ Treatment regimens } \\
\hline IMRT+CCRT & Reference & & Reference & \\
\hline IMRT alone & $2.826(1.362-5.864)$ & 0.005 & $2.538(1.335-4.825)$ & 0.004 \\
\hline \multicolumn{5}{|l|}{ MVA/Cox model } \\
\hline \multicolumn{5}{|c|}{ Treatment regimens } \\
\hline IMRT+CCRT & Reference & & Reference & \\
\hline IMRT alone & $1.934(1.085-3.450)$ & 0.025 & $2.511(1.240-5.088)$ & 0.011 \\
\hline \multicolumn{5}{|l|}{ Age } \\
\hline$\geq 60$ & $1.028(0.964-1.097)$ & 0.397 & $0.985(0.924-1.050)$ & 0.634 \\
\hline \multicolumn{5}{|l|}{ Sex } \\
\hline Male & Reference & & Reference & \\
\hline Female & $1.077(0.453-2.560)$ & 0.866 & $0.877(0.383-2.006)$ & 0.756 \\
\hline Body mass index & & 0.006 & & 0.036 \\
\hline$\leq 18.49$ & Reference & & Reference & \\
\hline $18.50-22.99$ & $0.535(0.206-3.650)$ & 0.200 & $0.699(0.241-2.032)$ & 0.511 \\
\hline $23.00-27.50$ & $0.355(0.138-0.914)$ & 0.031 & $0.439(0.149-1.300)$ & 0.137 \\
\hline$>27.50$ & $0.102(0.012-0.896)$ & 0.039 & $0.188(0.020-1.759)$ & 0.143 \\
\hline Education & & 0.166 & & 0.818 \\
\hline Unschooled & Reference & & Reference & \\
\hline Low & $0.822(0.341-1.981)$ & 0.663 & $0.738(0.279-1.951)$ & 0.540 \\
\hline Middle & $0.636(0.263-1.539)$ & 0.316 & $0.627(0.236-1.663)$ & 0.348 \\
\hline High & $0.526(0.180-1.533)$ & 0.239 & $0.917(0.302-2.787)$ & 0.878 \\
\hline \multicolumn{5}{|l|}{ Smoke } \\
\hline Yes & $1.600(0.789-3.330)$ & 0.209 & $1.299(0.641-2.632)$ & 0.468 \\
\hline No & Reference & & Reference & \\
\hline \multicolumn{5}{|l|}{ Drink } \\
\hline Yes & $0.914(0.389-2.147)$ & 0.837 & $1.048(0.452-2.430)$ & 0.912 \\
\hline No & Reference & & Reference & \\
\hline \multicolumn{2}{|c|}{ Charlson/Deyo comorbidity score } & 0.013 & & 0.738 \\
\hline 0 & Reference & & Reference & \\
\hline 1 & $1.518(0.830-2.776)$ & 0.176 & $1.238(0.665-2.304)$ & 0.500 \\
\hline$\geq 2$ & $3.310(1.313-8.346)$ & 0.011 & $1.053(0.306-3.619)$ & 0.902 \\
\hline VCA-IgA & & 0.198 & & 0.644 \\
\hline$<1: 80$ & Reference & & Reference & \\
\hline $1: 80-1: 320$ & 0.493(0.144-1.693) & 0.261 & $1.512(0.429-5.328)$ & 0.520 \\
\hline$\geq 1: 640$ & $0.713(0.233-2.180)$ & 0.553 & $1.624(0.344-7.669)$ & 0.541 \\
\hline EA-IgA & & 0.974 & & 0.176 \\
\hline$<1: 10$ & Reference & & Reference & \\
\hline $1: 10-1: 20$ & $0.495(0.216-1.135)$ & 0.097 & $0.647(0.215-1.947)$ & 0.438 \\
\hline$\geq 1: 40$ & $1.282(0.597-2.748)$ & 0.525 & $1.606(0.623-4.100)$ & 0.327 \\
\hline EBV-DNA & & 0.004 & & 0.003 \\
\hline $0-999$ & Reference & & Reference & \\
\hline $1000-9999$ & $2.111(0.827-5.391)$ & 0.118 & $3.193(1.296-7.868)$ & 0.012 \\
\hline $10000-99999$ & $2.790(1.174-6.632)$ & 0.020 & $2.909(1.204-7.029)$ & 0.018 \\
\hline $100000-$ & $3.004(1.055-8.552)$ & 0.039 & $3.393(1.175-9.795)$ & 0.024 \\
\hline Unmeasured & $1.604(0.769-3.343)$ & 0.207 & $1.927(0.907-4.092)$ & 0.088 \\
\hline \multicolumn{5}{|c|}{ Histology, WHO type } \\
\hline II & Reference & & Reference & \\
\hline III & $0.731(0.174-3.075)$ & 0.669 & $2.420(0.314-18.679)$ & 0.397 \\
\hline Tumor stage & & 0.072 & & 0.408 \\
\hline 1 & Reference & & Reference & \\
\hline 2 & $0.841(0.178-3.965)$ & 0.826 & $0.937(0.182-4.836)$ & 0.940 \\
\hline 3 & $0.538(0.120-2.413)$ & 0.418 & $0.725(0.145-3.628)$ & 0.696 \\
\hline 4 & $0.255(0.043-1.520)$ & 0.134 & $0.449(0.060-3.361)$ & 0.436 \\
\hline Node stage & & 0.070 & & 0.251 \\
\hline 0 & Reference & & Reference & \\
\hline 1 & $1.463(0.691-3.100)$ & 0.320 & $1.797(0.826-3.910)$ & 0.140 \\
\hline 2 & $1.267(0.556-2.898)$ & 0.572 & $1.411(0.587-3.396)$ & 0.442 \\
\hline 3 & $5.525(1.841-16.587)$ & 0.002 & $4.849(1.002-3.396)$ & 0.050 \\
\hline Clinical stage & & $<0.001$ & & $<0.001$ \\
\hline
\end{tabular}

\begin{tabular}{|c|c|c|c|c|}
\hline \multirow[t]{2}{*}{ Characteristics } & \multicolumn{2}{|l|}{ OS } & \multicolumn{2}{|l|}{ DFS } \\
\hline & $\mathrm{HR}(95 \% \mathrm{CI})$ & $\mathrm{P}$ & $\mathrm{HR}(95 \% \mathrm{CI})$ & $P$ value \\
\hline II & Reference & & Reference & \\
\hline III & $2.118(0.865-5.187)$ & 0.100 & $1.861(0.792-4.372)$ & 0.154 \\
\hline IVA-B & $7.218(2.890-18.028)$ & $<0.001$ & $4.668(3.579-10.851)$ & $<0.001$ \\
\hline Year of diagnosis & & 0.788 & & 0.681 \\
\hline $2002-2006$ & Reference & & Reference & \\
\hline 2007-2009 & $0.561(0.270-1.162)$ & 0.120 & $0.518(0.233-1.151)$ & 0.106 \\
\hline 2010-2013 & $0.868(0.412-1.827)$ & 0.710 & $1.029(0.484-2.186)$ & 0.941 \\
\hline \multicolumn{5}{|c|}{$\begin{array}{l}\text { * PSA/IPTW, propensity score analysis by inverse probability of treatment } \\
\text { weighting, was performed to estimated effects of regimens with observed } \\
\text { variables. }\end{array}$} \\
\hline \multicolumn{5}{|c|}{$\begin{array}{l}\text { Abbreviations: CCRT, concurrent chemotherapy; CI, confidence interval; DFS, } \\
\text { disease free survival; DNA, deoxyribonucleic acid; EBV, Epstein-Barr virus; IMRT, } \\
\text { intensity-modulated radiotherapy; MVA, multivariable analysis; OS, overall } \\
\text { survival. }\end{array}$} \\
\hline
\end{tabular}

\section{Survival based on EBV DNA}

To evaluate the prognostic value of EBV DNA in elderly patients with NPC, efficacy endpoints were determined for the EBV DNA-evaluable NPC patients. Compared with patients with low EBV DNA, patients with high EBV DNA levels (dichotomized by 4,000 copies $/ \mathrm{mL}$ ) showed worse OS and DFS (HR 2.701, 95\% CI 1.292-5.647, P = 0.008; HR $2.938,95 \%$ CI $1.412-6.113, \mathrm{P}=0.004$, respectively; Figure 2).

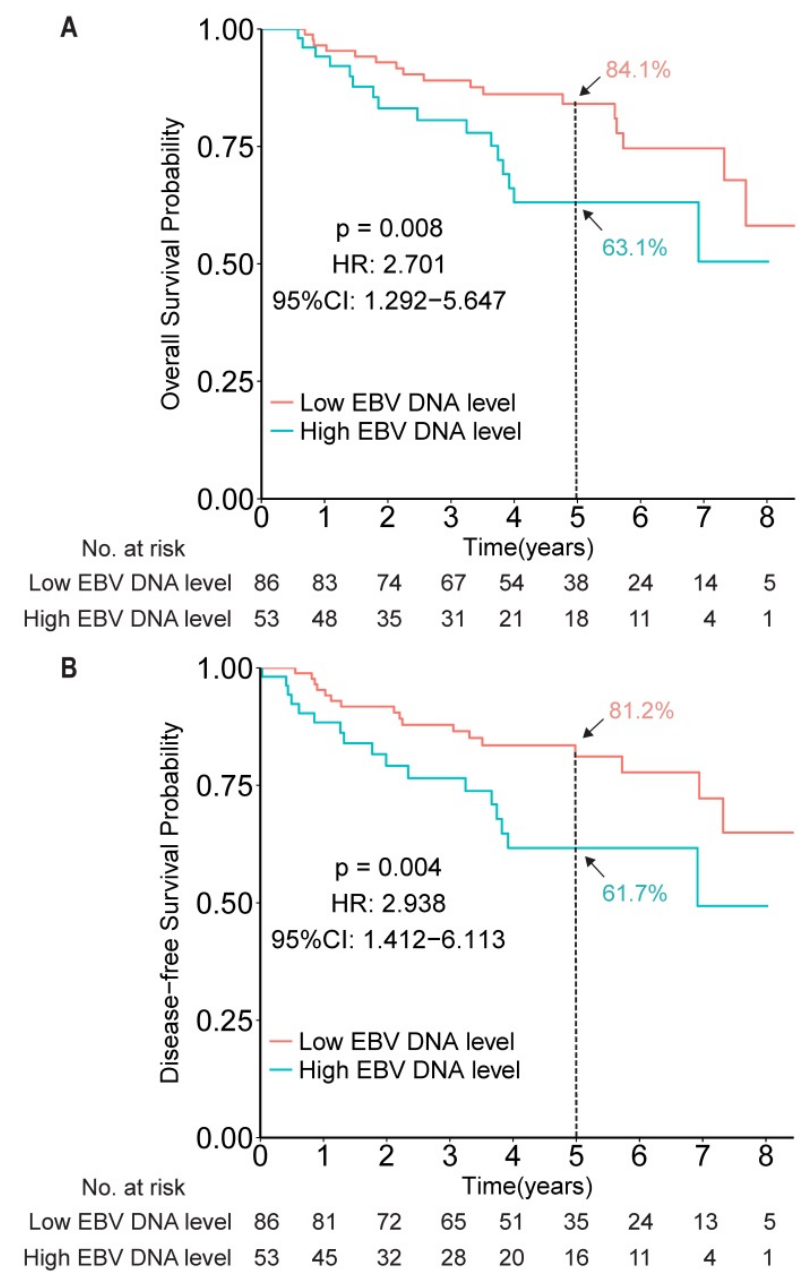

Figure 2. Kaplan-Meier survival curves for overall survival $(A)$ and disease-free survival (B) among patients with high and low EBV DNA levels in the measurable cohort, based on a cut-off of 4,000 copies $/ \mathrm{mL}$. 
After showing the prognostic role of EBV DNA levels in this elderly patient population, we evaluated its predictive role by examining the effect of EBV DNA levels on outcomes within a given treatment arm. After adjusting for other factors, the HR results indicated that IMRT plus CCRT improved both OS and DFS for patients with high EBV DNA levels (HR 3.316, 95\% CI 1.118-9.841, P = 0.031; HR 3.575, 95\% CI 1.236-10.339, $\mathrm{P}=0.019$, for OS and DFS, respectively), but not for patients with low EBV DNA levels (HR 2.131, 95\% CI 0.701-6.480, P = 0.182; HR 2.461, 95\% CI $0.807-7.504, \mathrm{P}=0.113$, for OS and DFS, respectively; Figure 3). However, no significant interaction effect between treatment group and EBV DNA level was observed $(\mathrm{P}=0.653)$.

\section{Treatment toxicity}

Table 4 shows the incidence of major acute and late toxicities due to treatments. For severe acute toxicity (graded 3 or higher), the incidence of leukopenia (22.3\% vs. $1.1 \%, \mathrm{P}<0.001)$, neutropenia (7.8\% vs. $0, \mathrm{P}=0.007)$, stomatitis $(39.8 \%$ vs. $16.8 \%, \mathrm{P}<$ $0.001)$, and emesis (5.8\% vs. $0, P=0.030)$ was significantly higher in the IMRT+CCRT group than in the IMRT-alone group, while no significant

A

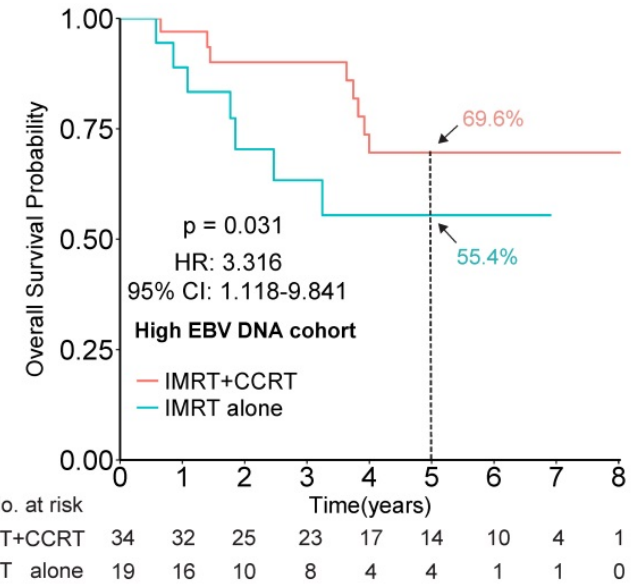

B

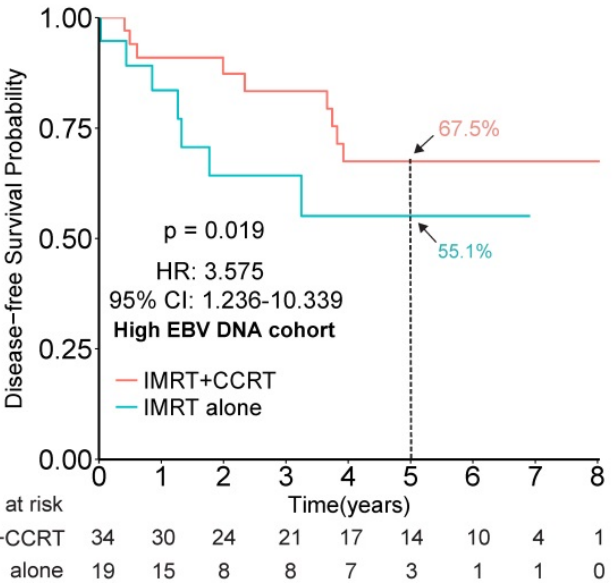

differences were found for thrombocytopenia, anaemia, or liver damage. Notably, one patient died from cardiopulmonary failure during treatment with CCRT plus IMRT. In total, there was a significant between-group difference in severe acute toxicity $(64.1 \%$ and $21.1 \%$ for the IMRT+CCRT and IMRT-alone groups, respectively, $\mathrm{P}<0.001)$. For severe late toxicity (grade 3 or higher), the total incidence rate of any severe late toxicity was $10.1 \%$ (20/198). In total, the incidence of severe late toxicity was comparable between the two groups $(10.7 \%$ and $9.5 \%$ for the IMRT+CCRT and IMRT-alone groups, respectively, $\mathrm{P}=0.818$ ).

\section{Sensitivity analysis}

Although similar results were revealed through different MVA models with various observational variables, including socioeconomic factors and a reliable biomarker, unmeasured confounders, i.e., the poor performance status of elderly patients, could potentially impact the prognostic HRs. Thus, based on the estimated treatment effects for survival in the entire cohort, sensitivity analysis was performed (Table 5). The gamma value was not small, indicating the robust results of the study analysis.

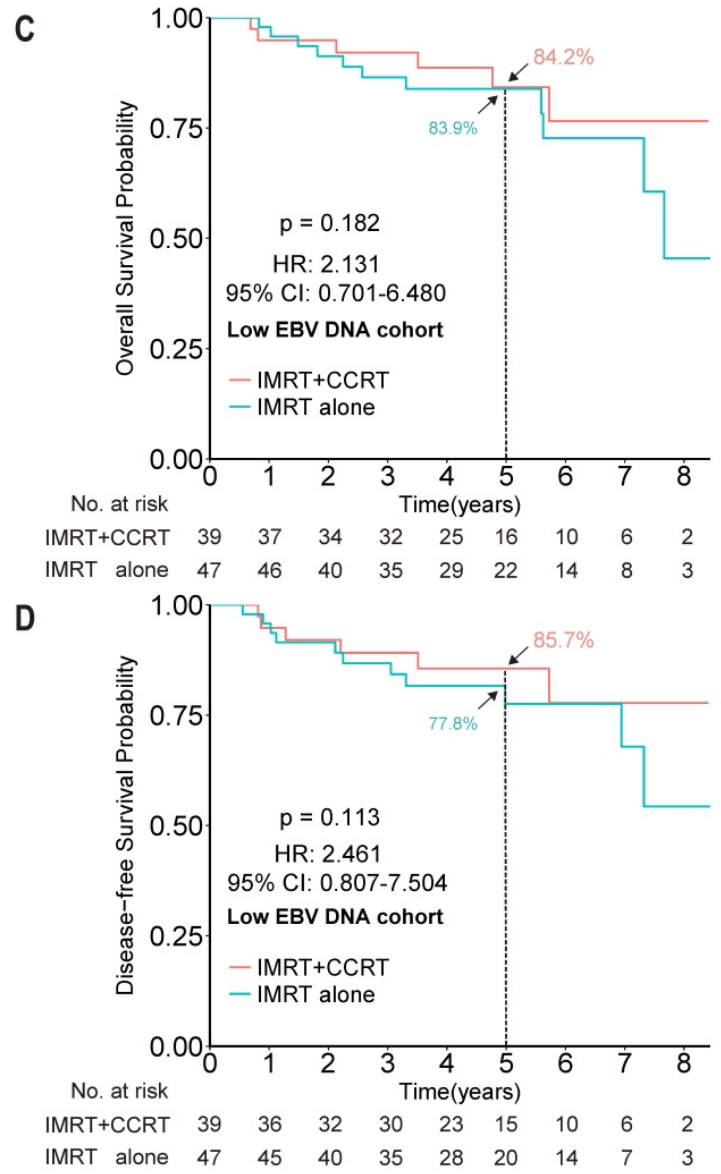

Figure 3. Kaplan-Meier survival curves for overall survival and disease-free survival between the IMRT+CCRT and IMRT-alone groups of elderly NPC patients with high EBV DNA levels (A and B, respectively) and of elderly patients with low EBV DNA levels (C and D, respectively). The cut-off EBV DNA level was selected as 4,000 copies/mL. Abbreviations: CCRT, concurrent chemotherapy; IMRT, intensity-modulated radiotherapy. 
Table 4. Elderly patients experienced severe (grade 3 or 4) toxicities by treatment regimens

\begin{tabular}{|c|c|c|c|c|c|c|c|}
\hline \multirow[t]{2}{*}{ Toxicity } & \multicolumn{3}{|c|}{ CCRT+IMRT (\%) } & \multicolumn{4}{|c|}{ IMRT alone (\%) } \\
\hline & $\begin{array}{l}\text { Grade } \\
3\end{array}$ & $\begin{array}{l}\text { Grade } \\
4\end{array}$ & All & $\begin{array}{l}\text { Grade } \\
3\end{array}$ & $\begin{array}{l}\text { Grade } \\
4\end{array}$ & All & $P$ value \\
\hline \multicolumn{8}{|l|}{$\begin{array}{l}\text { Acute toxicity } \\
\text { (hematological) }\end{array}$} \\
\hline Leukopenia & 20(19.4) & $3(2.9)$ & $23(22.3)$ & $1(1.1)$ & $0(0)$ & $1(1.1)$ & $<0.001$ \\
\hline Neutropenia & $7(6.8)$ & $1(1.0)$ & $8(7.8)$ & $0(0)$ & $0(0)$ & $0(0)$ & 0.007 \\
\hline Thrombocytopenia & $7(6.8)$ & $3(2.9)$ & 10(9.7) & $2(2.1)$ & $1(1.1)$ & $3(3.2)$ & 0.116 \\
\hline Anemia & $1(1.0)$ & $4(3.9)$ & $5(4.9)$ & $1(1.1)$ & $0(0)$ & $1(1.1)$ & 0.253 \\
\hline Liver damage & $1(1.0)$ & $0(0)$ & $1(1.0)$ & $0(0)$ & $0(0)$ & $0(0)$ & 1.000 \\
\hline \multicolumn{8}{|c|}{ (Non-hematological) } \\
\hline $\begin{array}{l}\text { Stomatitis } \\
\text { (mucositis) }\end{array}$ & $40(38.8)$ & $1(1.0)$ & $41(39.8)$ & $16(16.8)$ & $0(0)$ & $16(16.8)$ & $<0.001$ \\
\hline Emesis & $6(5.8)$ & $0(0)$ & $6(5.8)$ & $0(0)$ & $0(0)$ & $0(0)$ & 0.030 \\
\hline Total any & $57(55.3)$ & $8(7.8)$ & $66(64.1)^{*}$ & $19(20.0)$ & $1(1.0)$ & $20(21.1)$ & $<0.001$ \\
\hline \multicolumn{8}{|l|}{ Late toxicity } \\
\hline Ear (deafness/otitis) & $3(2.9)$ & $0(0)$ & $3(2.9)$ & $2(2.1)$ & 1(1.1) & $3(3.2)$ & 1.000 \\
\hline Brain & $4(3.9)$ & $0(0)$ & $4(3.9)$ & $1(1.1)$ & $0(0)$ & $1(1.1)$ & 0.415 \\
\hline $\begin{array}{l}\text { Subcutaneous } \\
\text { Fibrosis }\end{array}$ & $4(3.9)$ & $0(0)$ & $4(3.9)$ & $5(5.3)$ & $0(0)$ & $5(5.3)$ & 0.901 \\
\hline Trismus & $1(1.0)$ & $0(0)$ & $1(1.0)$ & $1(1.1)$ & $0(0)$ & $1(1.1)$ & 1.000 \\
\hline Total any & $11(10.7)$ & $0(0)$ & 11(10.7) & $8(8.4)$ & $1(1.1)$ & $9(9.5)$ & 0.778 \\
\hline
\end{tabular}

Table 5. Sensitivity analysis for HR of all-cause mortality adjusted for poor performance status.

\begin{tabular}{lllll}
\hline \multicolumn{2}{l}{$\begin{array}{l}\text { Prevalence of poor } \\
\text { performance status }\end{array}$} & Hazard ratio & \\
\hline IMRT+CCRT & $\begin{array}{l}\text { IMRT } \\
\text { alone }\end{array}$ & $\begin{array}{l}\text { Poor } \\
\text { performance }\end{array}$ & $\begin{array}{l}\text { Treatment (adjusted for } \\
\text { poor performance) }\end{array}$ & $95 \%$ CI \\
\hline 0.1 & 0.8 & 1.2 & 2.484 & $1.197-5.156$ \\
0.1 & 0.9 & 1.2 & 2.442 & $1.177-5.068$ \\
0 & 0.9 & 1.2 & 2.394 & $1.154-4.969$ \\
0 & 1 & 1.2 & 2.355 & $1.135-4.886$ \\
0.1 & 0.4 & 2 & 2.220 & $1.070-4.607$ \\
$\mathbf{0 . 1}$ & $\mathbf{0 . 5}$ & $\mathbf{2}$ & $\mathbf{2 . 0 7 2}$ & $\mathbf{0 . 9 9 8 - 4 . 3 0 0}$ \\
\hline
\end{tabular}

NOTE: Bold font indicates situations where poor performance status was strong enough to influence significance of rules (i.e. lower bound of 95\% CI crossed 1). Values based on multivariate analysis adjusted all-cause mortality HR of 2.826 (95\% CI, 1.362-5.864).

Abbreviations: CCRT, concurrent chemotherapy; CI, confidence interval; EBV, Epstein-Barr virus; IMRT, intensity-modulated radiotherapy.

\section{Discussion}

To the best of our knowledge, in the era of IMRT, this is the first study to assess plasma EBV DNA levels to examine the effect of adding CCRT to IMRT for elderly patients with NPC. According to pretreatment plasma EBV DNA, patients with high EBV DNA levels showed significantly worse survival than those with low EBV DNA levels (cut-off of 4,000 copies $/ \mathrm{mL}$ ). In the stratification analysis by EBV DNA level, CCRT retained survival benefits at high EBV DNA levels but not at low EBV DNA levels. Of note, CCRT was associated with higher rates of severe acute toxicity and led to one chemoradiotherapyrelated death in this study cohort.

Several previous studies have shown that CCRT can achieve reasonable survival and locoregional control in elderly NPC patients [6, 7]. However, most patients enrolled in these studies were treated with conventional 2DRT. Furthermore, the widespread use of IMRT has demonstrated excellent local control for NPC patients [1]; however, elderly patients who met the strict selection criteria accounted for only a small fraction of the patients evaluated in these reports. Therefore, in the era of IMRT, whether CCRT can significantly benefit elderly NPC patients is still unclear. In our study, based on a large cohort of elderly NPC patients receiving IMRT, the results showed that the addition of CCRT to IMRT for elderly patients did improve both OS and DFS.

The quantitative response of plasma EBV DNA to treatment has been investigated for prognostic risk assessment and treatment stratification [25]. Our analysis confirmed EBV DNA as an independent prognostic biomarker that can predict the prognosis of elderly NPC patients treated with IMRT. However, to the best of our knowledge, no previous study has explored whether a survival benefit could be obtained from CCRT for elderly patients receiving IMRT with different stratified levels of EBV DNA. As a result, among elderly patients with high EBV DNA levels, those who received IMRT plus CCRT showed a greater survival benefit in terms of both OS and DFS than did those who received IMRT alone. Nevertheless, elderly patients with low EBV DNA levels showed similar survival benefits in the two treatment regimens. Given the post hoc nature of these analyses and the absence of a significant interaction between treatment regimen and risk stratified by EBV DNA level, the results should be interpreted cautiously.

Compared to elderly patients in previous reports or large clinical trials, the elderly patients in our study seemed to have higher survival rates. The 5-year OS rates of the RT-alone and chemoradiotherapy groups reported by Zeng et al. were $40 \%$ and $62 \%$ [7]; Liu et al. reported rates of $39.3 \%$ and $54 \%$ [6]; the INT-0099 study reported rates of $37 \%$ and $67 \%$ [26]; the Hong Kong 9901 study reported rates of $64 \%$ and $68 \%$ [27]; and the PWHQEH-94 trial reported rates of $65.8 \%$ and $70.3 \%$, respectively [28]. However, the unadjusted analysis in our study showed rates of $76.2 \%$ and $73.8 \%$, respectively. In previous studies, the improvement in survival for NPC patients was partly attributable to IMRT [29-31]; thus, the survival rates observed in our study may have been due to the elderly patients receiving IMRT, which may have overcome the defects associated with conventional 2DRT planning. In other words, IMRT could deliver lethal doses to gross tumours while minimizing doses to adjacent tissues or organs at risk [32].

In terms of toxicity, there was one case of chemoradiotherapy-related death in the IMRT+CCRT group, and we observed that patients receiving IMRT 
plus CCRT suffered from higher rates of severe acute toxicity (leukopenia, stomatitis, and emesis) than did patients receiving IMRT alone, while the rates of severe late toxicity were similar in both groups, consistent with previous studies [7, 18]. However, in terms of severe acute toxicity from chemoradiotherapy and RT alone, Zeng et al. reported severe acute mucositis rates of $46 \%$ and $28.7 \%$, respectively, which were higher than the toxicity rates of $39.8 \%$ and $16.8 \%$, respectively, found in our study. The reason for this result might be that IMRT significantly reduced the incidence of side effects because of the anatomic complexity and benefits in accurately encompassing tumour targets.

We acknowledge that limitations exist in this study. First, the main limitation of this study is its retrospective nature. To minimize bias, we not only adopted different methods to confirm the analysis results but also performed sensitivity analysis to estimate the effects of unmeasured confounders to validate the robustness of the analysis. Second, the quantitative plasma EBV DNA cut-off of 4,000 copies/mL was selected based on our previous studies in the same endemic area. However, other cut-off have been suggested. Thus, we examined prespecified groups by order of magnitude and groups by cut-off to coordinate the analysis. However, whether combined IMRT and CCRT can provide a survival benefit to an optimal elderly population requires confirmation in further prospective randomized trials.

In conclusion, in the era of IMRT, this is the first study to assess plasma EBV DNA levels, and the results showed that there is a beneficial effect of adding CCRT to IMRT for elderly patients. The elderly patients with high EBV DNA levels showed significantly worse survival than did those with low EBV DNA levels. CCRT improved survival benefits for patients with high EBV DNA levels but not for those with low EBV DNA levels. Moreover, CCRT led to higher rates of severe acute toxicity. A prospective, well-designed clinical trial with a large cohort is required to confirm the efficacy of CCRT in elderly patients with NPC and to determine which subgroups can receive the greatest benefit from different treatment regimens.

\section{Supplementary Material}

Supplementary table.

http://www.jcancer.org/v09p3023s1.pdf

\section{Acknowledgements}

We thank Professor Qing Liu, Can-Hong Wen, and Lu Han for their help with this study and for their time and enthusiasm. This study was partly funded by the National Natural Science Foundation of China (Nos. 81472525, 81572665, 81672680, 81773162, 81572901, 31170151, and 81672872), the Science and Technology Planning Project of Guangdong Province, China (programme grants 2014A050503033, 2016A050502011，2014A020209024，2014B020212017, and 2015A020210046), and the Natural Science Foundation of Guangdong Province, China (programme grants 2017A030313866 and 2016A030311011). The authenticity of this article has been validated by uploading the key raw data onto the Research Data Deposit (RDD) public platform (www.researchdata.org.cn) with the approval RDD number as RDDA2018000609.

\section{Competing Interests}

The authors have declared that no competing interest exists.

\section{References}

1. Chua ML, Wee JT, Hui EP, Chan AT. Nasopharyngeal carcinoma. Lancet 2016; 387: 1012-1024.

2. Cao S-M, Simons MJ, Qian C-N. The prevalence and prevention of nasopharyngeal carcinoma in China. Chinese journal of cancer 2011; 30: 114.

3. Wee J, Ha TC, Loong S, Qian C. Is nasopharyngeal cancer really a" Cantonese cancer"? Chinese journal of cancer 2010; 29: 517-526.

4. Sarmiento MP, Mejia MB. Preliminary assessment of nasopharyngeal carcinoma incidence in the Philippines: a second look at published data from four centers. Chin J Cancer 2014; 33: 159-164.

5. Adham M, Kurniawan AN, Muhtadi AI et al. Nasopharyngeal carcinoma in Indonesia: epidemiology, incidence, signs, and symptoms at presentation. Chin J Cancer 2012; 31: 185-196.

6. Liu H, Chen QY, Guo L et al. Feasibility and efficacy of chemoradiotherapy for elderly patients with locoregionally advanced nasopharyngeal carcinoma: results from a matched cohort analysis. Radiat Oncol 2013; 8: 70.

7. Zeng $\mathrm{Q}$, Xiang $\mathrm{YQ}, \mathrm{Wu} \mathrm{PH}$ et al. A matched cohort study of standard chemo-radiotherapy versus radiotherapy alone in elderly nasopharyngeal carcinoma patients. PLoS One 2015; 10: e0119593.

8. Cao CN, Luo JW, Gao L et al. Concurrent chemotherapy for T4 classification nasopharyngeal carcinoma in the era of intensity-modulated radiotherapy. PLoS One 2015; 10: e0119101.

9. Sun $X$, Su S, Chen C et al. Long-term outcomes of intensity-modulated radiotherapy for 868 patients with nasopharyngeal carcinoma: an analysis of survival and treatment toxicities. Radiother Oncol 2014; 110: 398-403.

10. Lee L, Cheung WY, Atkinson E, Krzyzanowska MK. Impact of comorbidity on chemotherapy use and outcomes in solid tumors: a systematic review. J Clin Oncol 2011; 29: 106-117.

11. Lo YM, Chan LY, Chan AT et al. Quantitative and temporal correlation between circulating cell-free Epstein-Barr virus DNA and tumor recurrence in nasopharyngeal carcinoma. Cancer Res 1999; 59: 5452-5455.

12. Shao JY, Zhang Y, Li YH et al. Comparison of Epstein-Barr virus DNA level in plasma, peripheral blood cell and tumor tissue in nasopharyngeal carcinoma. Anticancer Res 2004; 24: 4059-4066.

13. Chan AT, Lo YM, Zee B et al. Plasma Epstein-Barr virus DNA and residual disease after radiotherapy for undifferentiated nasopharyngeal carcinoma. J Natl Cancer Inst 2002; 94: 1614-1619.

14. Chen WH, Tang LQ, Wang FW et al. Elevated levels of plasma D-dimer predict a worse outcome in patients with nasopharyngeal carcinoma. BMC Cancer 2014; 14: 583

15. Leung SF, Zee B, Ma BB et al. Plasma Epstein-Barr viral deoxyribonucleic acid quantitation complements tumor-node-metastasis staging prognostication in nasopharyngeal carcinoma. J Clin Oncol 2006; 24: 5414-5418.

16. Zhao C, Han F, Lu LX et al. [Intensity modulated radiotherapy for local-regional advanced nasopharyngeal carcinoma]. Ai Zheng 2004; 23: 1532-1537.

17. Chen L, Hu CS, Chen XZ et al. Concurrent chemoradiotherapy plus adjuvant chemotherapy versus concurrent chemoradiotherapy alone in patients with locoregionally advanced nasopharyngeal carcinoma: a phase 3 multicentre randomised controlled trial. Lancet Oncol 2012; 13: 163-171.

18. Chen QY, Wen YF, Guo L et al. Concurrent chemoradiotherapy vs radiotherapy alone in stage II nasopharyngeal carcinoma: phase III randomized trial. J Natl Cancer Inst 2011; 103: 1761-1770. 
19. Cox JD, Stetz J, Pajak TF. Toxicity criteria of the Radiation Therapy Oncology Group (RTOG) and the European Organization for Research and Treatment of Cancer (EORTC). Int J Radiat Oncol Biol Phys 1995; 31: 1341-1346.

20. Therasse P, Arbuck SG, Eisenhauer EA et al. New guidelines to evaluate the response to treatment in solid tumors. European Organization for Research and Treatment of Cancer, National Cancer Institute of the United States, National Cancer Institute of Canada. J Natl Cancer Inst 2000; 92: 205-216.

21. Austin PC. The performance of different propensity score methods for estimating marginal hazard ratios. Stat Med 2013; 32: 2837-2849.

22. Gayat E, Resche-Rigon M, Mary JY, Porcher R. Propensity score applied to survival data analysis through proportional hazards models: a Monte Carlo study. Pharm Stat 2012; 11: 222-229.

23. Qin J, Ning J, Liu H, Shen Y. Maximum Likelihood Estimations and EM Algorithms with Length-biased Data. J Am Stat Assoc 2011; 106: 1434-1449.

24. Wang CY. Robust sandwich covariance estimation for regression calibration estimator in Cox regression with measurement error. Statistics \& Probability Letters 1999; 45: 371-378.

25. Leung SF, Chan KC, Ma BB et al. Plasma Epstein-Barr viral DNA load at midpoint of radiotherapy course predicts outcome in advanced-stage nasopharyngeal carcinoma. Ann Oncol 2014; 25: 1204-1208.

26. Al-Sarraf M, LeBlanc M, Giri PG et al. Chemoradiotherapy versus radiotherapy in patients with advanced nasopharyngeal cancer: phase III randomized Intergroup study 0099. J Clin Oncol 1998; 16: 1310-1317.

27. Lee AW, Tung SY, Chua DT et al. Randomized trial of radiotherapy plus concurrent-adjuvant chemotherapy vs radiotherapy alone for regionally advanced nasopharyngeal carcinoma. J Natl Cancer Inst 2010; 102: 1188-1198.

28. Chan AT, Leung SF, Ngan RK et al. Overall survival after concurrent cisplatin-radiotherapy compared with radiotherapy alone in locoregionally advanced nasopharyngeal carcinoma. J Natl Cancer Inst 2005; 97: 536-539.

29. Lee N, Harris J, Garden AS et al. Intensity-modulated radiation therapy with or without chemotherapy for nasopharyngeal carcinoma: radiation therapy oncology group phase II trial 0225. J Clin Oncol 2009; 27: 3684-3690.

30. Peng G, Wang T, Yang KY et al. A prospective, randomized study comparing outcomes and toxicities of intensity-modulated radiotherapy vs. conventional two-dimensional radiotherapy for the treatment of nasopharyngeal carcinoma. Radiother Oncol 2012; 104: 286-293.

31. Lee AW, Ng WT, Chan LL et al. Evolution of treatment for nasopharyngeal cancer--success and setback in the intensity-modulated radiotherapy era. Radiother Oncol 2014; 110: 377-384.

32. Kam MK, Chau RM, Suen J et al. Intensity-modulated radiotherapy in nasopharyngeal carcinoma: dosimetric advantage over conventional plans and feasibility of dose escalation. Int J Radiat Oncol Biol Phys 2003; 56: $145-157$ 